\title{
The utility of stem cells in pediatric urinary bladder regeneration
}

\author{
Philip M lannaccone $e^{1,2,3,4}$, Vasil Galat ${ }^{1,3,4}$, Matthew I Bury ${ }^{1,3,5,6}$, Yongchao C Ma ${ }^{1,2,3,7}$ and Arun K Sharma $a^{1,3,5,6,8,9}$
}

Pediatric patients with a neurogenic urinary bladder, caused by developmental abnormalities including spina bifida, exhibit chronic urological problems. Surgical management in the form of enterocystoplasty is used to enlarge the bladder, but is associated with significant clinical complications. Thus, alternative methods to enterocystoplasty have been explored through the incorporation of stem cells with tissue engineering strategies. Within the context of this review, we will examine the use of bone marrow stem cells and induced pluripotent stem cells (iPSCs), as they relate to bladder regeneration at the anatomic and molecular levels. The use of bone marrow stem cells has demonstrated significant advances in bladder tissue regeneration as multiple aspects of bladder tissue have been recapitulated including the urothelium, bladder smooth muscle, vasculature, and peripheral nerves. iPSCs, on the other hand, have been well characterized and used in multiple tissue-regenerative settings, yet iPSC research is still in its infancy with regards to bladder tissue regeneration with recent studies describing the differentiation of iPSCs to the bladder urothelium. Finally, we examine the role of the Sonic Hedgehog signaling cascade that mediates the proliferative response during regeneration between bladder smooth muscle and urothelium. Taken together, this review provides a current, comprehensive perspective on bladder regeneration.

$\mathbf{T}$ he primary function of the urinary bladder is to act as a distensible storage repository for urine under conditions of low internal bladder pressure and to evacuate to completion under facultative control. The bladder is a highly elastic, hollow, muscular organ that is relatively simplistic in structure comprising a tri-layered architecture consisting of urothelium-lined lumen surrounded by serosal and bladder smooth muscle layers interspersed with vascular and neural networks. Upon severe insult in the form of trauma, cancer, or developmental defects, the end-stage neurogenic bladder can demonstrate a loss of storage capacity and bladder compliance followed by partial bladder-emptying. Detrimental sequelae of the neurogenic bladder may include chronic urinary tract infections, incontinence, and the formation of bladder and renal calculi accompanied by renal deterioration leading to eventual renal failure (1). In order to alleviate the consequences of these clinical manifestations, end-stage neurogenic bladders undergo surgical intervention in the form of bladder augmentation enterocystoplasty, considered as the "gold-standard" treatment within the urological field. Although this surgical procedure alleviates certain physiological perturbations of the neurogenic bladder, it is accompanied by many short- and long-term complications (2). In light of these shortcomings, tissue-engineering strategies utilizing an armamentarium of synthetic materials in combination with an assortment of stem cells that seek to delineate alternative strategies to bladder augmentation enterocystoplasty. Within the context of this review, we will examine the utility of stem cells, specifically with regards to the potential contribution of exogenous cells in a bladderregenerative setting, along with an overview of Hedgehog signaling as it pertains to bladder regeneration.

\section{BONE MARROW CELLS AND THEIR APPLICATIONS IN BLADDER REGENERATION}

The marrow-containing long bones of humans contain specific microenvironments that are stratified into vascular and hematopoietic niches (3-5). Bone marrow (BM) provides the body with mature blood cells derived from undifferentiated stem and progenitor cells through a complex cascade of physiological events. The bone marrow cavity is also lined with stroma at the marrow/periosteum interface in which hematopoietic and non-hematopoietic stem cells reside and receive microenvironmental cues for mobilization and differentiation. The stromal cell system is further divided into cell populations including fibroblasts and mesenchymal stem cells (MSCs). MSCs reside within the marrow cavity, maintain a level of self-renewal, and give rise to cells that can differentiate into various tissue lineages. These populations

\footnotetext{
Ann and Robert H. Lurie Children's Hospital of Chicago, Chicago, Illinois; ${ }^{2}$ Department of Pediatrics, Northwestern University Feinberg School of Medicine, Chicago, Illinois; ${ }^{3}$ Developmental Biology Program, Stanley Manne Children's Research Institute, Chicago, Chicago, Illinois; ${ }^{4}$ Department of Pathology, Northwestern University Feinberg School of Medicine, Chicago, Illinois; ${ }^{5}$ Division of Pediatric Urology, Ann and Robert H. Lurie Children's Hospital of Chicago, Chicago, Illinois; ${ }^{6}$ Simpson Querrey Institute, Northwestern University, Chicago, Illinois; ${ }^{7}$ Department of Neurology, Northwestern University Feinberg School of Medicine, Chicago, Illinois; ${ }^{8}$ Department of Urology, Northwestern University Feinberg School of Medicine, Chicago, Illinois; ${ }^{9}$ Department of Biomedical Engineering, Northwestern University McCormick School of Engineering, Evanston, Illinois. Correspondence: Arun K. Sharma (asharma@luriechildrens.org)

Received 18 May 2017; accepted 7 September 2017; advance online publication 8 November 2017. doi:10.1038/pr.2017.229
} 
are functionally and phenotypically defined as osteocytes, adipocytes, and chondrocytes, as well as a host of other cells (6).

MSCs represent a heterogenous cell population initially characterized from the bone marrow, yet can be isolated from multiple tissues from within the body $(7,8)$. MSCs embody a potent plasticity whose attributes can be harnessed for a vast number of tissue-engineering approaches. Within the context of bladder regeneration, a former study described the phenotypic and physiological similarities between adult MSCs and bladder smooth muscle cells (SMCs) (9). This study describes the characterization of several cellular contractile proteins, demonstrating that unstimulated MSCs have a similar contractile protein profile as their bladder SMC counterparts. These proteins included smooth muscle alpha actin, smoothelin, transgelin, calponin, and vimentin. Further data demonstrated that MSCs and bladder SMCs possess similar contractile machinery and can respond to agonist stimulation with physiologically indistinguishable contractile responses without the use of cytokine induction for the MSCs (9). Through in vitro intracellular $\mathrm{Ca}^{2+}$ release assays, carbachol-stimulated MSCs and bladder SMCs resulted in no significant difference between both groups with regards to the magnitude of increase $(5.8 \pm 0.8$ and $8.6 \pm 2.5$ (Relative Fluorescence Units), respectively, $P<0.0001$ ), indicating the similar physiological features between the cell types (9). A second study described the use of adult MSCs in a nude rat urinary bladder augmentation model (10). These MSCs were also compared with bladder SMCs that had been seeded upon a novel, synthetic elastomeric scaffold (poly 1,8 octanediol-cocitrate; POC). MSC-seeded POC scaffolds maintained high levels of protein expression of smooth muscle markers including caldesmon, elastin, calponin, and $\gamma$-actin, and further displayed smooth muscle bundle formation by immunochemical staining while preventing bladder stone formation (10). Quantitative histological data also revealed a statistically significant increase in the muscle-to-collagen ratios at 10 weeks post augmentation compared with bladder SMCseeded POC scaffolds. The muscle-to-collagen ratios in MSCseeded POC constructs were $\sim 1: 1$, where it was $\sim 3: 7$ and 2:8 in bladder smooth muscle and unseeded POC constructs, indicating high collagen levels that inhibit the elastic nature of the bladder leading to severe physiological complications including bladder fibrosis. The typical muscle-to-collagen ratio found in the bladder across multiple species is $~ 1: 1$ (11). These data suggest that MSCs can serve as an alternative cell source for potentially diseased bladder SMCs. These studies represent stepping stones toward research endeavors that may delineate the further roles of MSCs in bladder regeneration, potentially in the context of other cell types.

The other cellular component of the bone marrow is the multipotent hematopoietic stem/progenitor cell (HSPC). The bone marrow stroma physically supports the differentiation of primitive HSPCs that comprises a hierarchy of cells that have the ability to either self-renew or terminally differentiate into daughter cells with more specified functions (12-15). A specific fraction of HSPCs, namely CD34+ HSPCs and its subfractions, has the unique ability to induce robust tissue vascularization. A major impediment of current tissueengineering strategies is to delineate means in which grafts that are structurally large in nature can be adequately vascularized, especially at the core of tissue grafts where oxygen and nutrient exchange is poor. Several studies have utilized CD34+ HSPCs to jumpstart tissue angiogenesis (1619). Kawamoto et al. describe the intramyocardial transplantation of human CD34+ HSPCs into nude mice following myocardial infarction (20). Data from this study demonstrate that capillary density was significantly higher in treated groups vs. controls, and this was accompanied by decreases in percent tissue fibrosis. Echocardiographic fractional shortening on day 28 was significantly higher in the CD34+ cell group as the regional wall motion score was also significantly better-preserved in treated groups (20). Even as the utility of $\mathrm{CD} 34+\mathrm{HSPCs}$ increases in the context of tissue-engineering multiple organ systems, its use has been limited in the bladder tissue-engineering arena.

Recently, a novel research undertaking demonstrated the unique ability of bone-marrow-derived MSCs in combination with CD34+ HSPCs to recapitulate bladder tissue in vivo (21). The most compelling aspect of this study was that the bone marrow cells utilized for bladder augmentation surgery were procured from pediatric spina bifida (SB) patients diagnosed with a neurogenic bladder. In vitro analyses of MSCs showed that pediatric SB patients retained cellular multipotentiality with the ability to undergo standard trilineage differentiation into adipocytes, osteocytes, and chondrocytes combined with nearly identical proliferative capacity and immunophenotyping profiles when compared with adult and pediatric control MSC counterparts (21). Further cDNA microarray profiling of SB-derived MSCs revealed significant gene signature overlap with the differential expression of 129 unique genes when compared with adult and pediatric control MSCs. However, the most striking aspect of this study was the in vivo analyses. Following partial bladder cystectomy, nude rats were augmented with various combinations of cell-seeded POC scaffolds that included MSCs, CD34+ HSPCs, or the MSC/CD34+ HSPC combination from either adult, pediatric, or pediatric SB bone marrow donors. Quantitative post-augmentation data revealed an approximately ninefold increase in percent vasculature in regenerated tissue when utilizing the MSC/CD34+ HSPC construct in the SB donor group compared with unseeded controls. Immunostaining indicated that the blood vessels that circumvented the entirety of the bladder graft were derived from the seeded cells. Similar values were reflective in other donor groups demonstrating that the combination of these two cell types was pivotal in the vascularization of the aggregate graft, including the core where graft failure is often initiated because of the lack of tissue oxygen perfusion by blood. The MSC/CD34+ HSPC combination also provided the impetus for peripheral nerve regeneration-a key component that has been lacking in previous bladder 


\section{Review | Iannaccone et al.}

regeneration studies and that is absolutely crucial for proper bladder contraction/expansion cycles. The peripheral nerve regeneration was attributed specifically to the CD34+ HSPC component, as this cell population has been shown to express nerve growth factor and its receptors and was in-growth from the remaining bladder tissue (21). The data demonstrate that the developmental defects associated with pediatric SB had no negative effects on bone-marrow-derived stem/progenitor cell functionality and may be a suitable source of autologous cells for bladder regeneration.

Another study exemplified the use of depleted whole bone marrow cells in bladder regeneration (22). Data from this study indicated that bladder reconstitution occurs more rapidly using a morphologically fibroblastic component of whole BM cells that have been seeded upon small intestinal submucosa compared with sham or unseeded control animals in a rat bladder augmentation model. These cell-seeded scaffolds maintained high levels of protein expression of known muscle markers such as smooth muscle alpha actin. This study further demonstrated the utility of bone marrow cells for bladder regeneration. This and other studies exemplify the existence and utility of cellular constituents of the bone marrow that have pivotal roles in the regenerative process of the bladder, although those mechanistic pathways are not completely understood (22). Preliminary data from our laboratory indicate a role of the Hedgehog signaling pathway in bladder tissue within the confines of bone-marrow-derived stem cell bladder regeneration.

\section{HEDGEHOG SIGNALING IN BLADDER REGENERATION}

Signal transduction pathways including the Hedgehog/ Patched/GLI (HH/PTCH/GLI) pathway are important in normal development, congenital anomalies, and in cancers (23-28). These pathways augment the developmental induction established by regulating genes and often interact with each other (29). Sonic Hedgehog ( $\mathrm{SHH}$ ) signal and GLI are critical to prostate, bladder smooth muscle, and motor neuron development (30-33). Activation of the SHH signal transduction pathway is essential for the specification of ventral spinal cord progenitor domains and the differentiation of spinal motor neurons (33). Smooth muscle in the urinary bladder develops from primitive mesenchyme directed by urothelial signals $(34,35)$. Proliferative response in regeneration of bladder smooth muscle and urothelium requires $\mathrm{SHH}$ signal crosstalk between urothelium and stroma $(36,37)$. Proliferative responses are abrogated in the absence of GLI1. On the other hand, the overexpression of GLI1 as a consequence of Suppressor of Fused (SuFu) knockout mutation in mice results in neural tube closure defects (38) as in SB. This is the opposite of the general concept that overexpression of the signaling pathway results in cancer, whereas reduced activity of the pathway results in severe birth defects. This is likely due to ventralization of the neural tube at a critical developmental juncture. Regardless, exploring the manipulation of the $\mathrm{SHH}$ pathway activity could provide fruitful avenues of approach to both proliferative capacity during regeneration or prevention of the posterior closure defects that lead to bladder dysfunction in the first place.

SHH and GLI1 are necessary for urothelial regeneration following chemical or bacterial injury. Shin et al. induced injury and initiated a regenerative cycle in mice with an uropathogenic strain of Escherichia coli or with protamine sulfate exposure (36). The bacteria resulted in $72 \%$ increase in Ki67-positive epithelial cells and $28 \%$ of stromal cells in $24 \mathrm{~h}$. Chemical exposure resulted in less epithelial proliferation and essentially no stromal response. The injury-induced response was primarily in basal urothelial cells. A primary response to $\mathrm{SHH}$ is increasing GLI1 mRNA and both were increased following injury. GLI1 expression was limited to stroma, although the SHH expression was in all layers. Urothelial cells were capable of forming multilayered spheres that developed into cysts in which the outer stromal cells express $\mathrm{Ck} 5$ and $\mathrm{SHH}$, whereas the inner lining cells did not. The evidence suggests that there are resident multipotent stem cells in the basal urothelium. Importantly, it also suggests that pluripotent cells are capable of regenerating bladder (39). The advantage to this, of course, is that all other cell types necessary to bladder function could be present and integrated. It is clear that $\mathrm{SHH}$ signaling is critical to this outcome.

$\mathrm{HH} / \mathrm{PTCH} / \mathrm{GLI}$ is a complex signal transduction pathway involving reversal of negative regulation of a seven-span transmembrane protein, Smoothened (SMO) by the receptor Patched (PTCH1, 2), following stimulation by the $\mathrm{HH}$ ligand (including $\mathrm{SHH}$ ). In the absence of ligand signal, $\mathrm{PTCH}$ destabilizes SMO and prevents it from trafficking to the membrane from intracytoplasmic vesicles. The signaling center is located in the primary cilium of the cell and in the presence of ligand $(\mathrm{SHH})$ the $\mathrm{PTCH}$ complex internalizes preventing PTCH from locking SMO intracellularly (40-42). PTCH internalization is regulated in part by the ubiquitinating enzymes SMURF1 and SMURF2 (ref. 43). Three transcription factor family members, GLI1, GLI2, and GLI3, mediate signaling. Without $\mathrm{SHH}$ ligand transcriptional regulatory activity of GLI1 is repressed by complexing with $\mathrm{SuFu}$, preventing GLI1 from moving to the nucleus. The presence of $\mathrm{SHH}$ ligand induces $\mathrm{SMO}$ to localize in the primary cilium and represses SuFu leading to the release of GLI1 from the primary cilium. The GLI/SuFu complex is regulated in a cilium tip compartment whose architecture is in part regulated by Kif7 (ref. 33). In the presence of ligand, PTCH is endocytosed and it does not inhibit SMO. Without repression from PTCH, the GLI transcription factors localize to the nucleus and regulate target gene expression $(45,46)$. A significant disease burden is associated with dysregulation of this signal transduction pathway. In general, increased somatic signaling activity is associated with cancers, whereas decreased germline signaling activity is associated with birth defects except for neural tube closure defects.

GLI1 is transcriptionally activated by GLI2 and noncanonical signaling can be induced by cMYC, KRAS, TGF $\beta$ WNT, or beta-catenin. Our sequencing a number of years ago 
and public reference sequence data show six highly conserved GLI-binding sites in the large first intron of the GLI1 gene (25). Although all three GLI family members can bind GLI-binding sites, how the target is discriminated by them is not known. Moreover, GLI1 most commonly activates, but can also repress expression of target genes, whereas in general GLI2 activates gene targets, GLI2 alternate splice forms can repress gene targets, and GLI3 represses gene targets. $(24,26)$. How this is accomplished and what drives the decision to activate or repress is not known. There is strong evidence suggesting that GLI genes autoregulate in a positive feedback loop (47-49). The negative regulation of this feedback loop could be provided by GLI3, translational repression, or by the repressive effects of the lncRNA, GLIlas (GLI1 antisense long non-coding RNA) (50). Translational repression is the result of an riboneucleoprotein complex involving Quaking, a tumor suppressor (51). IncRNA genes are frequently associated with developmentally relevant genes and inhibit transcription of genes through binding of the regulatory regions or by other mechanisms.

As discussed above, GLI1 is a transcription factor mediating SHH signal critical to regulating cell division and apoptosis (23-25,52). In bladders of mutant mice lacking GLI1 the proliferative response to both bacterial and chemical injury was greatly reduced in both the stromal and urothelial compartments. Thus, epithelial SHH induction of GLI1 signaling in stroma leads to a combined stromal/epithelial proliferative response during regeneration. Cross-tissue layer signal transduction in this pathway has been shown in bone cells as well $(37,53)$. Important regulation of SHH signaling occurs between tissue layers with crosstalk been the mesenchyme and epithelium. The role of such signaling in the regenerating urothelium is suspected, but is not well understood. Such activity has been described in the context of normal prostate development and prostate cancer, and increasing information supports the role of such crosstalk in increasing efficiency of engraftment and function of the regenerated urothelium $(27,30,37,54)$. The role of extracellular matrix in regeneration and tissue engineering has been emphasized. There is a strong effort underway to establish
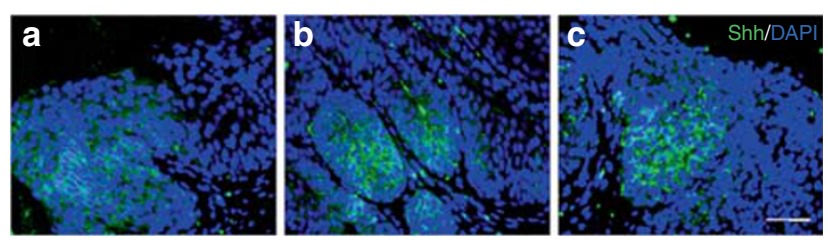

Figure 1. SHH expression following bladder augmentation. Nude rats were partially cystectomized and augmented with human bone marrow cell-seeded POC scaffolds. (a) Normal pediatric MSC/CD34+ HSPCs. (b) Pediatric spina bifida MSC/CD34+ HSPCs. (c) Adult MSC/CD34+ HSPCs. Shh expression was limited to aspects of the regenerating urothelium in each donor type, whereas native bladder tissue had sparse expression of SHH. Subjective preliminary data from this study suggest a yet to be determined role of SHH in regenerating bladder urothelium. Staining was performed 4 weeks post augmentation. Magnification $\times 40$; bar $=100 \mu \mathrm{m}$. Image courtesy of the Sharma Laboratory. HSPC, hematopoietic stem/progenitor cell; POC, poly 1,8 octanediol-co-citrate. the characteristics of bioscaffolds that will enhance ECM-like interactions with stem cells (55). Understanding the role of signaling pathways particularly the SHH pathway in bladder regeneration will add to the ability to predict extracellular states required for optimal stem cell interaction for tissue engineering (56).

However, the role of Shh within other bladder tissue injury situations, including bladder augmentation surgery, has not been elucidated. It has been shown that bone marrow stemcell-seeded scaffolds can achieve functional bladder regeneration in a small animal model, but the molecular players have not been completely elucidated. Signaling pathways interact with each other modulating the signaling outcomes $(57,58)$. This results in outputs from combined signals that are different from the individual signal output. The ability to modulate signal output in this way is critical to normal development. In particular, there is crosstalk between the Wnt and Hedgehog signaling pathways (59); family members of the Wnt signaling pathway including TCF3/4, Wnt10a, and Fzd5 are highly expressed in the regenerating urothelium (but to a much lesser degree in native urothelium) during the regenerative program following bladder augmentation (21). We speculated that SHH may be present in the regenerating urothelium following bladder augmentation. Figure 1 demonstrates the expression of $\mathrm{SHH}$ in regenerating the urothelium following augmentation in $\mathrm{MSC} / \mathrm{CD} 34+\mathrm{POC}-$ seeded scaffolds from three different donor types. As an alternative for utilizing cell sources for bladder tissue regeneration, the use of SHH small molecule stimulators/ inhibitors may be useful tools to promote regeneration in situations where it is warranted such as direct bladder trauma.

\section{PLURIPOTENT-CELL-BASED BIOLOGICALLY ACTIVE PROS- THETIC GRAFTS}

Human embryonic stem cells and induced pluripotent stem cells (iPSCs) provide a uniquely scalable source of functionally differentiated cells. iPSCs represent an ever-evolving class of cells that can be derived in a non-invasive, autologous manner and possess the potential to be utilized in a wide range of clinical settings. Highly plastic in nature, iPSCs are regarded as prime candidates for tissue-engineering strategies, especially in the context of biological grafts. Biologically active prosthetic grafts comprising living cells maintain the flexibility and capacity to develop and differentiate into specific tissue types, which is pivotal for complete repair in the pediatric patient population. With respect to bladder regeneration, the most significant advantage of iPSC technology is the potential of producing the aforementioned cell types in a bladder-regenerative setting that will eventually comprise functional bladder tissue in an autologous manner. Moreover, iPSCs are the only possible source for neural cells as they potentiate peripheral and motor nerve regeneration, as they currently cannot be obtained from adult tissues. Thus, the derivation and utilization of iPSCs could allow for highcapacity bladder tissue regeneration leading to a fully 


\section{Review Iannaccone et al.}

functional bladder. Although the advent of iPSCs has been heralded in many fields, there has been very limited use of iPSCs in the context of bladder tissue regeneration with minimal peer-reviewed studies currently published in the literature. As it has been previously demonstrated that different bone marrow cell populations can act as surrogate cell sources for bladder tissue, iPSCs may be used to obtain these primitive cell types or attempt to derive bladder cell types, such as the urothelium, directly (60). Urothelium, a type of transitional epithelium, develops through a definitive endoderm stage. Accordingly, endodermal markers have been characterized during iPSC differentiation that include Gata416, Sox17, FOXA2, as well as markers typical for epithelial differentiation such as CK8/18, ZO-1, and E-Cadherin (61). Although expression of important urothelium-specific markers including the family of uroplakin and cytokeratin proteins was detected, the bladder urothelium represents a multilayered structure consisting of basal cells, intermediate cells, and apical (or umbrella) cells. However, the in vivo functionality of iPSC-derived cells remains to be assayed. A study by Osborn et al. describes the in vitro derivation of the bladder urothelium from human embryonic stem cells and iPSCs (62). Through the culturing of cells with specialized urothelium-specific differentiating media, the group was able to show that human embryonic stem cells and iPSCs could terminally differentiate into bladder urothelium through the expression of the transcription factors IFR1, GATA4, and GET1 accompanied by uroplakin expression (62). Another differentiation protocol that produces urothelium with expression markers characteristic for apical including uroplakins (UPIb, UPII, and UPIIIa) is described in a study by Kang et al. (63). A more recent study by Wang et al. shows that iPSCs can be reprogrammed into the smooth muscle component of the bladder sphincter and theoretically, by extension, into components of the bladder wall itself (64). Following the periurethral injection of rats exhibiting stress urinary incontinence with smooth muscle precursor cells derived from human iPSCs, the authors were able to show that there was a statistically significant increase in bladder leak point pressure (LPP) compared with control, shamsaline, iPSC-pSMC, and Epi-iPSC-pSMC animals by LPP measurements. These novel approaches may provide suitable future alternative options when other cell types are unavailable. The use of iPSCs is still in its infancy with regards to pediatric bladder regeneration and needs to be heavily scrutinized going forward. However, precursor cells including MSCs and vascular progenitor cells are still imperative for bladder-regenerative purposes. In the event that MSCs are not donor-ready, iPSC-derived MSCs and vascular progenitor cells may serve as suitable alternatives in the interim.

Clinical trials to evaluate the safety of the product are underway for human embryonic stem cell derivatives for agerelated macular degeneration, type I diabetes, spinal cord injury, myocardial infarction, and Parkinson's disease. The first clinical trial for iPSCs initiated by the pioneering team led by Takahashi for age-related macular degeneration and led to the seminal paper describing the first safe clinical use of IPSc-derived sheets of retinal pigmented epithelial cells aimed at sight restoration in a human patient $(65,66)$.

\section{MESENCHYMAL STEM CELL DERIVATION FROM HUMAN IPSCS}

Adult MSCs derived from varying donor types or tissues may possess variability in cell quality and quantity. As the derivation of MSCs from iPSCs (iMSCs) has been demonstrated, the idea of utilizing iMSCs in therapy is gaining interest in a vast array of regenerative tissue settings (67-69). In comparison with MSCs derived from an adult source, iMSCs are more homogenous, possess an increased proliferative capacity, and are less intrusive to obtain. For instance, Simpson et al. has shown significant similarities in gene expression of iMSC compared with BM-MSC, whereas the proliferation capacity of iPSC-MSC was almost twice as that of BM-MSC (69). The enhanced proliferative effect may be explained by the epigenetic rejuvenation during reprogramming (70).

The majority of protocols used for the derivation of MSCs from human iPSCs utilize adherent culture conditions as described by Boyd et al. (71). A novel method of iPSC mesenchymal differentiation has demonstrated the isolation of a multipotential progenitor at the mesenchymoangioblast stage and the growth of colony-forming units in a semisolid medium (72,73). With this approach, the derived iMSCs produce more potent immunomodulatory effects than the MSCs from the adult source (74). The self-renewing characteristics of iPSCs allow for the introduction of genetic modifications, for instance, favorable alterations of immunomodulatory functions that enhance homing of iPSC-derived MSCs following transplantations. As iMSCs may have unlimited supply, they have advantage for the chronic conditions, with a prolonged period of treatment or when a substantial amount of cells is needed as in the bladderregenerative setting.

\section{SCAFFOLD PRE-VASCULARIZATION}

Ischemia contributes to cellular death and is frequently observed at the early onset of scaffold transplantation, especially in the context of large grafts $(75,76)$. There are two main approaches that could lead to an increase in the viability and physiological potential of implanted stem cells following cell transplantation. This can be accomplished either by inducing host angiogenesis with pro-angiogenic growth factors, including those released from synthetic platforms or from the pre-vascularization of the graft (7781). Scaffold pre-vascularization techniques primarily consist of manipulating endothelial cells, and occasionally SMCs or pericytes for vessel stabilization, before construct implantation (82). Most efforts at microvasculature and capillary tissue-engineering work involve the use of allogeneic sources such as human umbilical vein endothelial cells and MSCs. The autologous collection of endothelial cells is performed by mobilizing rare populations of endothelial progenitor cells 
from peripheral blood, or cells obtained by invasive means like autologous bone-marrow-derived MSCs. The invasiveness of autologous endothelial cells harvesting and low proliferation rate have restricted its broader clinical application. iPSC-derived endothelial cells provide a sufficiently expandable, translatable patient-specific cell source that may enhance rapid vascularization of engineered construct, while maximizing biocompatibility and minimizing rejection risk. In addition to oxygenation, vascular cells provide a humoral support for functional maturation of neighboring cellular components, such as neurons and peripheral nerves (83).

\section{ENDOTHELIAL CELL AND SMOOTH MUSCLE CELL DERIVA- TION FROM hPSCS}

The ability of human ESCs to give rise to endothelial cells is widely documented (84). Initially, the strategies used to coax cells into endothelial cells included in vitro differentiation by embryoid bodies or by culturing cells on OP9 stromal cells, but as these initial strategies were developed, alternatives have become more effective. The induction of iPSCs by WNT signaling modulation demonstrates superior iPSC endothelial cell-derived quality in terms of proliferation, marker stability, and essential functions (85). The iPS-ECs retain 98\% expression of CD31+/CD34+/CD144+ at passage 5, accumulate LcLDL, build a tubular network and proliferate until passage 18. To induce differentiation, iPSCs were cultured in the presence of GSK3 inhibitor, which yields up to $40 \%$ endothelial $\mathrm{CD} 34+/ \mathrm{CD} 31+$ progenitors (86). Other bladder tissue-engineering strategies have been employed that utilize endothelial progenitor cells to vascularize potential grafts. Chen et al. assess the utility of autologous, genetically modified porcine endothelial progenitor cells that overexpress vascular endothelial growth factor (VEGF; VEGF OX) to establish functional blood conduits in a porcine bladder cystectomy model (87). VEGF OX endothelial-cell-seeded bladder acellular matrix grafts demonstrated histologically normal bladder tissue. This was accompanied by an approximately fivefold increase in microvessel density (MVD) 6 months post surgery compared with 1-month-old animals and a 1.5-fold increase in MVD compared with unseeded bladder acellular matrix graft at the 6-month time
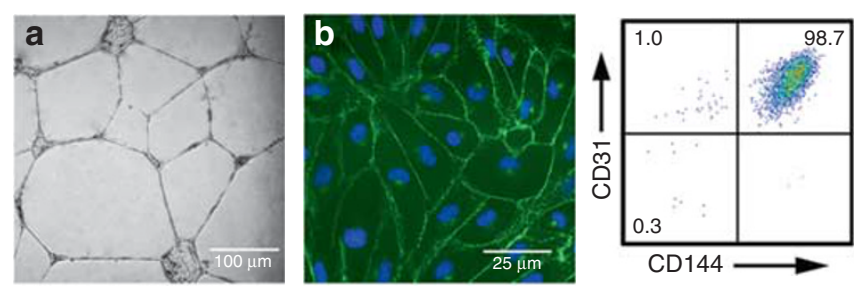

Figure 2. Phenotypic and functional characterization of endothelial cells obtained from iPSCs (induced endothelial cells; iECs). (a) Tube formation assay, (b) immunostaining: VE-Cadherin expression (green), and cells were co-stained with 4',6-diamidino-2-phenylindole, dihydrochloride (blue); (c) Fluorescence-activated cell sorting demonstrated that $>99 \%$ of the cells coexpress VE-Cadherin (CD144) and PECAM1 (CD31). Image courtesy of the Galat Laboratory. IPSC, induced pluripotent stem cell. point. Although there was a moderate increase in the MVD at 6 months compared with control, the level of bladder tissue vascularization could be vastly improved in future studies. This may be accomplished by increasing the number of endothelial progenitor cells seeded onto the graft as well as the addition of microvascular patterning in the form of an arborizing pattern, which would best utilize the surface area of the graft when seeded (88). This would be typically accomplished with synthetic grafts such as POC that can undergo topographical manipulation at the macro and micro levels.

As bladder SMCs are a vital component for the architectural and functional foundation of the bladder, exogenous, nonpathological sources to be utilized as alternate replacement cells would be highly desired. In the case of SB, for example, bladder SMCs may be deleteriously affected by disease progression. The aforementioned iPSC-derived CD34 $+/ \mathrm{CD} 31+$ progenitor population can produce both endothelial cells and SMCs in the proper culture environments (89-91) Here, we show the derivation of endothelial cells from iPSCs (Figure 2) that could be potentially utilized in a bladderregenerative setting. Further treatment of these cells with TGF- $\beta$ enhanced SMC differentiation from these endothelial progenitors, whereas the TGF- $\beta$ signaling inhibitor SB431542 promoted endothelial cell generation (92). Combined, these strategies provide viable solutions in generating cells that can be used for tissue-regenerative medicine applications for the bladder.

\section{CONCLUSION}

Approaches for bladder tissue engineering have encompassed the use of myriad cell types along with multiple iterations of biologic and synthetic scaffolds with moderate success. Autologous bone marrow stem and progenitor cells have provided great insight into the utility of these cells in a bladder-regenerative setting especially in the context of developmental defects that may affect bladder tissue as in the case of SB. Although novel in approach, studies such as these are limited by the quality and quantity of cells that may be obtained, and these are crucial for graft development and in vivo sustainability. iPSCs may provide a robust, alternative, and autologous option so that the bladder tissue components including the musculature, urothelium, serosa, and vascular and neural networks may be regenerated at gross anatomic and functional levels. However, in order to better understand these complex tissue cellularity relationships in the regenerating bladder, one must examine molecular events that ultimately will determine the fate of grafts. The SHH pathway has demonstrated its pleiotropic effects in a variety of other organ systems, but has demonstrated its regenerative potential in the context of bladder regeneration. Future studies may specifically target the SHH pathway with agonist/antagonist strategies in order to modulate bladder regeneration. Finally and most importantly, as bladder regeneration endeavors have evolved over time, the majority of these studies have focused their attention on clinical scenarios that are applicable 
to the adult population. As anatomy and physiology vary quite differently from the adult to pediatric populations, greater focus needs to be applied on the pediatric population with the hopes of a future translational component. This is evident by the dearth of bladder-regenerative studies that focus on the pediatric population.

\section{ACKNOWLEDGMENTS}

A.K.S is supported by NIH grant R01DK109539, The Hartwell Foundation, and the Stanley Manne Children's Research Institute. V.G. was supported in part by NIH grant RC1HL100168 and the Stanley Manne Children's Research Institute. P.M.I. was supported by the George M. Eisenberg Foundation for Charities. V.G. and P.M.I. were also supported by the Illinois Regenerative Medicine Institute. Y.C.M. is supported by NIH grant numbers R01NS094564, The Hartwell Foundation, the Chicago Biomedical Consortium, Cure SMA Foundation, and Whitehall Foundation. Y.C.M. is the Ann Marie and Francis Klocke M.D. Research Scholar supported by the Joseph and Bessie Feinberg Foundation.

Disclosure: The authors declare no conflict of interest.

\section{REFERENCES}

1. Ab E, Dik P, Klijn AJ, van Gool JD, de Jong TP. Detrusor overactivity in spina bifida: how long does it need to be treated? Neurourol Urodyn 2004;23:685-8.

2. Bankhead RW, Kropp BP, Cheng EY. Evaluation and treatment of children with neurogenic bladders. J Child Neurol 2000;15:141-9.

3. Morrison SJ, Scadden DT. The bone marrow niche for haematopoietic stem cells. Nature 2014;505:327-4.

4. Sugiyama T, Nagasawa T. Bone marrow niches for hematopoietic stem cells and immune cells. Inflamm Allergy Drug Targets 2012;11:201-6.

5. Anthony BA, Link DC. Regulation of hematopoietic stem cells by bone marrow stromal cells. Trends Immunol 2014;35:32-7.

6. Augello A, De Bari C. The regulation of differentiation in mesenchymal stem cells. Hum Gene Ther 2010;21:1226-38.

7. Abreu SC, Antunes MA, Xisto DG, et al. Bone marrow, adipose, and lung tissue-derived murine mesenchymal stromal cells release different mediators and differentially affect airway and lung parenchyma in experimental asthma. Stem Cells Transl Med 2017;6:1557-67.

8. Deng Y, Zhang Y, Ye L, et al. Umbilical cord-derived mesenchymal stem cells instruct monocytes towards an IL10-producing phenotype by secreting IL6 and HGF. Sci Rep 2016;6:37566.

9. Sharma AK, Fuller NJ, Sullivan RR, et al. Defined populations of bone marrow derived mesenchymal stem and endothelial progenitor cells for bladder regeneration. J Urol 2009;182 (4 Suppl): 1898-905.

10. Sharma AK, Hota PV, Matoka DJ, et al. Urinary bladder smooth muscle regeneration utilizing bone marrow derived mesenchymal stem cell seeded elastomeric poly(1,8-octanediol-co-citrate) based thin films. Biomaterials 2010;31:6207-17.

11. Caione P, Capozza N, Zavaglia D, Palombaro G, Boldrini R. In vivo bladder regeneration using small intestinal submucosa: experimental study. Pediatr Surg Int 2006;22:593-9.

12. Seita J, Weissman IL. Hematopoietic stem cell: self-renewal versus differentiation. Wiley Interdiscip Rev Syst Biol Med 2010: 640-53.

13. Takakura N, Watanabe T, Suenobu S, et al. A role for hematopoietic stem cells in promoting angiogenesis. Cell 2000;102:199-209.

14. Sahoo S, Klychko E, Thorne T, et al. Exosomes from human CD34(+) stem cells mediate their proangiogenic paracrine activity. Circ Res 2011;109:724-8.

15. Losordo DW, Henry TD, Davidson C, et al. Intramyocardial, autologous CD34+ cell therapy for refractory angina. Circ Res 2011;109:428-36.

16. Pozzoli O, Vella P, Iaffaldano G, et al. Endothelial fate and angiogenic properties of human $\mathrm{CD} 34+$ progenitor cells in zebrafish. Arterioscler Thromb Vasc Biol 2011;31:1589-97.
17. Asahara T, Murohara T, Sullivan A, et al. Isolation of putative progenitor endothelial cells for angiogenesis. Science 1997;275:964-7.

18. Tateishi-Yuyama E, Matsubara H, Murohara T, et al. Therapeutic angiogenesis for patients with limb ischaemia by autologous transplantation of bone-marrow cells: a pilot study and a randomised controlled trial. Lancet 2002;360:427-35.

19. Kudo FA, Nishibe T, Nishibe M, Yasuda K. Autologous transplantation of peripheral blood endothelial progenitor cells $(\mathrm{CD} 34+)$ for therapeutic angiogenesis in patients with critical limb ischemia. Int Angiol 2003;22: 344-8.

20. Kawamoto A, Iwasaki H, Kusano K, et al. CD34-positive cells exhibit increased potency and safety for therapeutic neovascularization after myocardial infarction compared with total mononuclear cells. Circulation 2006;114:2163-9.

21. Sharma AK, Bury MI, Fuller NJ, et al. Cotransplantation with specific populations of spina bifida bone marrow stem/progenitor cells enhances urinary bladder regeneration. Proc Natl Acad Sci USA 2013;110:4003-8.

22. Kanematsu A, Yamamoto S, Iwai-Kanai E, et al. Induction of smooth muscle cell-like phenotype in marrow-derived cells among regenerating urinary bladder smooth muscle cells. Am J Pathol 2005;166:565-753.

23. Yoon W, Gallant M, Lamm ML, et al. Non-canonical regulation of the Hedgehog mediator GLI1 by c-MYC in Burkitt Lymphoma. Mol Cancer Res 2013;11:604-15.

24. Yoon JW, Gilbertson R, Iannaccone S, Iannaccone P, Walterhouse D. Defining a role for Sonic hedgehog pathway activation in desmoplastic medulloblastoma by identifying GLI1 target genes. Int J Cancer 2009;124: 109-9.

25. Iannaccone PM, Taylor R, Long J, et al. Intronic regulation of human GLI1 DNA by cis DNA elements and epigenetic marks. FASEB J 2016;30:1180.1.

26. Villavicencio EH, Walterhouse DO, Iannaccone PM. The sonic hedgehog-patched-gli pathway in human development and disease. Am J Hum Genet 2000;67:1047-55.

27. Walterhouse DO, Lamm ML, Villavicencio E, Iannaccone PM. Emerging roles for hedgehog-patched-Gli signal transduction in reproduction. Biol Reprod 2003;69:8-14.

28. Robbins DJ, Fei DL, Riobo NA. The Hedgehog signal transduction network. Sci Signal 2012: 5 re6.

29. Balsara Z.R., Li X. Sleeping beauty: awakening urothelium from its slumber. Am J Physiol Renal Physiol 2017: F732-43.

30. Lamm ML, Catbagan WS, Laciak RJ, et al. Sonic hedgehog activates mesenchymal Glil expression during prostate ductal bud formation. Dev Biol 2002;249:349-66.

31. Yu M, Gipp J, Yoon JW, Iannaccone P, Walterhouse DO, Bushman W. Sonic hedgehog-responsive genes in the fetal prostate. J Biol Chem 2009;284:5620-9.

32. Motoyama J, Milenkovic L, Iwama M, Shikata Y, Scott MP, Hui CC. Differential requirement for Gli2 and Gli3 in ventral neural cell fate specification. Dev Biol 2003;259:150-61.

33. Jessell TM. Neuronal specification in the spinal cord: inductive signals and transcriptional codes. Nat Rev Genet 2000;1:20-9.

34. Peyton CC, Burmeister D, Petersen B, Andersson KE, Christ G. Characterization of the early proliferative response of the rodent bladder to subtotal cystectomy: a unique model of mammalian organ regeneration. PLoS ONE 2012;7:e47414.

35. Shiroyanagi Y, Liu B, Cao M, et al. Urothelial sonic hedgehog signaling plays an important role in bladder smooth muscle formation. Differentiation 2007;75:968-77.

36. Shin K, Lee J, Guo N, et al. Hedgehog/Wnt feedback supports regenerative proliferation of epithelial stem cells in bladder. Nature 2011;472:110-4.

37. Zunich SM, Douglas T, Valdovinos M, et al. Paracrine sonic hedgehog signalling by prostate cancer cells induces osteoblast differentiation. Mol Cancer 2009;8:12. 
38. Svärd J, Heby-Henricson K, Persson-Lek M, et al. Genetic elimination of Suppressor of fused reveals an essential repressor function in the mammalian Hedgehog signaling pathway. Dev Cell 2006;10:187-97.

39. Snow-Lisy DC, Diaz EC, Bury MI, et al. The role of genetically modified mesenchymal stem cells in urinary bladder regeneration. PLoS ONE 2015;10:e0138643.

40. Briscoe J, Therond PP. The mechanisms of Hedgehog signalling and its roles in development and disease. Nat Rev Mol Cell Biol 2013;14:416-29.

41. Hui CC, Angers S. Gli proteins in development and disease. Annu Rev Cell Dev Biol 2011;27:513-37.

42. Wong SY, Reiter JF. The primary cilium at the crossroads of mammalian hedgehog signaling. Curr Top Dev Biol 2008;85:225-60.

43. Yue S, Tang LY, Tang Y, et al. Requirement of Smurf-mediated endocytosis of Patched1 in sonic hedgehog signal reception. Elife 2014; 12:3:e02555.

44. He M, Subramanian R, Bangs F, et al. The kinesin-4 protein Kif7 regulates mammalian Hedgehog signalling by organizing the cilium tip compartment. Nat Cell Biol 2014;16:663-72.

45. Gerhardt C, Leu T, Lier JM, Rüther U. The cilia-regulated proteasome and its role in the development of ciliopathies and cancer. Cilia 2016;10: 14.

46. Kasper M, Schnidar H, Neill GW, et al. Selective modulation of Hedgehog/GLI target gene expression by epidermal growth factor signaling in human keratinocytes. Mol Cell Biol 2006;26:6283-98.

47. Dahmane N, Lee J, Robins P, Heller P, Ruiz I, Altaba A. Activation of the transcription factor Gli1 and the Sonic hedgehog signalling pathway in skin tumours. Nature 1997;389:876-1.

48. Dai P, Akimaru H, Tanaka Y, Maekawa T, Nakafuku M, Ishii S. Sonic Hedgehog-induced activation of the Gli1 promoter is mediated by GLI3. J Biol Chem 1999;274:8143-52.

49. Regl G, Neill GW, Eichberger T, et al. Human GLI2 and GLI1 are part of a positive feedback mechanism in basal cell carcinoma. Oncogene 2002;21:5529-39.

50. Villegas VE, Rahman MF, Fernandez-Barrena MG, et al. Identification of novel non-coding RNA-based negative feedback regulating the expression of the oncogenic transcription factor GLI1. Mol Oncol 2014;8:912-26.

51. Lakiza O, Frater L, Yoo Y, et al. STAR proteins quaking-6 and GLD-1 regulate translation of the homologues GLI1 and tra-1 through a conserved RNA 3'UTR-based mechanism. Dev Biol 2005;287:98-110.

52. Yoon JW, Kita Y, Frank DJ, et al. Gene expression profiling leads to identification of GLI1-binding elements in target genes and a role for multiple downstream pathways in GLI1-induced cell transformation. J Biol Chem 2002;277:5548-55.

53. Zunich SM, Valdovinos M, Douglas T, Walterhouse D, Iannaccone P, Lamm ML. Osteoblast-secreted collagen upregulates paracrine Sonic hedgehog signaling by prostate cancer cells and enhances osteoblast differentiation. Mol Cancer 2012;11:30.

54. Iannaccone PM, Lamm MLG, Yoon JW, Holmgren R, Ahlgren S, Lakiza O, Walterhouse DO, The Hedgehog Signaling Pathway in Development and Disease. In "Inborn Errors of Development: The Molecular Basis of Clinical Disorders of Morphogenesis." Epstein CJ et al. (eds). 2nd edn, 2008

55. Shridhar A, Gillies E, Amsden BG, Flynn LE. Composite Bioscaffolds Incorporating Decellularized ECM as a Cell-Instructive Component Within Hydrogels as In Vitro Models and Cell Delivery Systems, Totowa, NJ: Humana Press, 1-26.

56. Simoes IN, Vale P, Soker S, et al. Acellular urethra bioscaffold: decellularization of whole urethras for tissue engineering applications. Sc Rep 2017;7:41934.

57. Vert G, Chory J. Crosstalk in cellular signaling: background noise or the real thing? Dev Cell 2011;21:985-1.

58. Nishi H, Demir E, Panchenko AR. Crosstalk between signaling pathways provided by single and multiple protein phosphorylation sites. J Mol Biol 2015;427:511-20.
59. Song L, Li ZY, Liu WP, Zhao MR. Crosstalk between Wnt/beta-catenin and Hedgehog/Gli signaling pathways in colon cancer and implications for therapy. Cancer Biol Ther 2015;16:1-7.

60. Moad M, Pal D, Hepburn AC, et al. A novel model of urinary tract differentiation, tissue regeneration, and disease: reprogramming human prostate and bladder cells into induced pluripotent stem cells. Eur Urol 2016;64:753-61.

61. Galat V, Malchenko S, Galat Y, et al. A model of early human embryonic stem cell differentiation reveals inter- and intracellular changes on transition to squamous epithelium. Stem Cells Dev 2012;21:1250-63.

62. Osborn SL, Thangappan R, Luria A, Lee JH, Nolta J, Kurzrock EA. Induction of human embryonic and induced pluripotent stem cells into urothelium. Stem Cells Transl Med 2014;3:610-9.

63. Kang M, Kim HH, Han YM. Generation of bladder urothelium from human pluripotent stem cells under chemically defined serum- and feeder-free system. Int J Mol Sci 2014;15:7139-57.

64. Wang Z, Wen Y, Li YH, et al. Smooth muscle precursor cells derived from human pluripotent stem cells for treatment of stress urinary incontinence. Stem Cells Dev 2016;25:453-61.

65. Trounson A, DeWitt ND. Pluripotent stem cells progressing to the clinic. Nat Rev Mol Cell Biol 2016;1017:194-200.

66. Mandai M, Watanabe A, Kurimoto Y, et al. Autologous induced stemcell-derived retinal cells for macular degeneration. N Engl J Med 2017;376:1038-46.

67. Sheyn D, Ben-David S, Shapiro G, et al. Human induced pluripotent stem cells differentiate into functional mesenchymal stem cells and repair bone defects. Stem Cells Transl Med 2016;5:1447-60.

68. Lian Q, Zhang Y, Liang X, Gao F, Tse HF. Directed differentiation of human-induced pluripotent stem cells to mesenchymal stem cells. Methods Mol Biol 2016;1416:289-98.

69. Simpson DL, Wehman B, Galat Y, et al. Engineering patient-specific valves using stem cells generated from skin biopsy specimens. Ann Thorac Surg 2014;98:947-54.

70. Frobel J, Hemeda H, Lenz $M$, et al. Epigenetic rejuvenation of mesenchymal stromal cells derived from induced pluripotent stem cells. Stem Cell Rep 2014;3:414-22.

71. Boyd NL, Robbins KR, Dhara SK, West FD, Stice SL. Human embryonic stem cell-derived mesoderm-like epithelium transitions to mesenchymal progenitor cells. Tissue Eng Part A 2009;15:1897-907.

72. Galat V, Galat Y, Perepitchka M, Jennings LJ, Iannaccone PM, Hendrix MJ. Transgene reactivation in induced pluripotent stem cell derivatives and reversion to pluripotency of induced pluripotent stem cell-derived mesenchymal stem cells. Stem Cells Dev 2016;25:1060-72.

73. Vodyanik MA, Yu J, Zhang X, et al. A mesoderm-derived precursor for mesenchymal stem and endothelial cells. Cell Stem Cell 2010;7:718-29.

74. Kimbrel EA, Kouris NA, Yavanian GJ, et al. SMesenchymal stem cell population derived from human pluripotent stem cells displays potent immunomodulatory and therapeutic properties. Stem Cells Dev 2014;23: 1611-24.

75. Serbo JV, Gerecht S. Vascular tissue engineering: biodegradable scaffold platforms to promote angiogenesis. Stem Cell Res Ther 2013;4:8.

76. Osborn SL, So M, Hambro S, Nolta JA, Kurzrock EA. Inosculation of blood vessels allows early perfusion and vitality of bladder grafts-implications for bioengineered bladder wall. Tissue Eng 2015 Part A 21:1906-5.

77. Zhou L, Yang B, Sun C, et al. Coadministration of platelet-derived growth factor-BB and vascular endothelial growth factor with bladder acellular matrix enhances smooth muscle regeneration and vascularization for bladder augmentation in a rabbit model. Tissue Eng Part A 2013;19:264-76.

78. Loai $\mathrm{Y}$, Yeger $\mathrm{H}, \mathrm{Coz} \mathrm{C}$, et al. Bladder tissue engineering: tissue regeneration and neovascularization of HA-VEGF-incorporated bladder acellular constructs in mouse and porcine animal models. J Biomed Mater Res A 2010;94:1205-5.

79. Sharma AK, Bury MI, Fuller NJ, et al. Growth factor release from a chemically modified elastomeric poly(1,8-octanediol-co-citrate) thin film promotes angiogenesis in vivo. J Biomed Mater Res A 2012;100:561-70. 
80. Noguchi R, Nakayama K, Itoh $\mathrm{M}$, et al. Development of a threedimensional pre-vascularized scaffold-free contractile cardiac patch for treating heart disease. J Heart Lung Transplant 2016;35:137-45.

81. Wu Y, Cao L, Xia L, et al. Evaluation of osteogenesis and angiogenesis of icariin in local controlled release and systemic delivery for calvarial defect in ovariectomized rats. Sci Rep 2017;7:5077.

82. Bae H, Puranik AS, Gauvin R, et al. Building vascular networks. Sci Transl Med 2012;4:1 60ps23.

83. Schwartz MP, Hou Z, Propson NE, et al. Human pluripotent stem cellderived neural constructs for predicting neural toxicity. Proc Natl Acad Sci USA 2015;112:12516-21.

84. Kokudo T, Suzuki Y, Yoshimatsu Y, Yamazaki T, Watabe T, Miyazono K. Snail is required for TGFbeta-endothelial-mesenchymal transition of embryonic stem cell-derived endothelial cells. J Cell Sci 2008;121:3317-24.

85. Lian X, Bao X, Al-Ahmad A, et al. Efficient differentiation of human pluripotent stem cells to endothelial progenitors via small-molecule activation of WNT signaling. Stem Cell Rep 2014;3:804-16.

86. Galat Y, Dambaeva S, Elcheva I, et al. Cytokine free directed differentiation of hPSC efficiently produces hemogenic endothelium with lymphoid potential. Stem Cell Res Ther 2017;8:67.
87. Chen BS, Xie H, Zhang SL, et al. Tissue engineering of bladder using vascular endothelial growth factor gene-modified endothelial progenitor cells. Int J Artif Organs 2011;34:1137-46.

88. Sun JF, Phung T, Shiojima I, et al. Microvascular patterning is controlled by fine-tuning the Akt signal. Proc Natl Acad Sci UAA 2005;102: $128-33$.

89. Bai H, Gao Y, Arzigian M, Wojchowski DM, Wu WS, Wang ZZ. BMP4 regulates vascular progenitor development in human embryonic stem cells through a Smad-dependent pathway. J Cell Biochem 2010;109: 363-74.

90. Patsch C, Challet-Meylan L, Thoma EC, et al. Generation of vascular endothelial and smooth muscle cells from human pluripotent stem cells. Nat Cell Biol 2015;17:994-1003.

91. Yang L, Geng Z, Nickel T, et al. Differentiation of human inducedpluripotent stem cells into smooth-muscle cells: two novel protocols. PLoS ONE 2016;11:e0147155.

92. James D, Nam HS, Seandel M, et al. Expansion and maintenance of human embryonic stem cell-derived endothelial cells by TGFbeta inhibition is Id1 dependent. Nat Biotechnol 2010;28:161-6. 